\title{
Antibody response early after experimental infection with Mycobacterium avium subspecies paratuberculosis in dairy calves
}

\author{
Rienske A. R. Mortier, Herman W. Barkema, Maria E. Negron, Karin Orsel, Robert Wolf, and Jeroen De Buck ${ }^{1}$ \\ Department of Production Animal Health, University of Calgary, 3330 Hospital Drive NW, T2N 4N1, Calgary, Alberta, Canada
}

\begin{abstract}
Serological testing in the early stages of Johne's disease has been successful using specific antigens and inhouse ELISA. However, the use of a commercial ELISA has not been evaluated shortly after Mycobacterium avium subspecies paratuberculosis (MAP) infection, nor has it been determined whether this serological response is age or dose dependent. Fifty-six calves were randomly allocated to challenge groups ( 5 per group) and a negative control group. Calves were inoculated orally on 2 consecutive days at 2 wk or at $3,6,9$, or 12 mo. Within each age group, 5 calves received either a high or low dose of MAP. Using a commercial ELISA, antibody responses were detected in $42 \%$ of the inoculated calves and were present in all age and dose groups (except for the 6-mo low-dose group). Antibody response profiles differed among individual calves; persistent as well as peak and bimodal peak responses existed. Calves inoculated at 12 mo were ELISA positive within 4.5 mo after inoculation, whereas those inoculated at younger ages took longer to become ELISA positive. Furthermore, calves inoculated with a high dose of MAP more often became ELISA positive than low-dose calves when inoculated at a younger age. In conclusion, a dose-dependent antibody response was detected by ELISA in a larger proportion of calves than expected soon after inoculation.
\end{abstract}

Key words: paratuberculosis, antibody response, ELISA, dairy calf

\section{INTRODUCTION}

Paratuberculosis or Johne's disease (JD) is a chronic enteritis of ruminants caused by Mycobacterium avium ssp. paratuberculosis (MAP; Tiwari et al., 2006). The major effects are reduced milk yield (Benedictus et al., 1987; Kudahl et al., 2004), premature culling, and reduced slaughter value (Chi et al., 2002). The incubation period for JD in cattle ranges from 2 to $10 \mathrm{yr}$ (Whitlock

Received March 14, 2014.

Accepted May 22, 2014.

${ }^{1}$ Corresponding author: jdebuck@ucalgary.ca and Buergelt, 1996); the most common clinical signs are chronic, untreatable diarrhea, and wasting (Tiwari et al., 2006).

Infection with MAP is initially controlled by a T helper 1 (Th1) immune response, which is a cellular immune response (Stabel, 2000). At approximately the onset of clinical symptoms, this response shifts to a $\mathrm{T}$ helper 2 (Th2) immune response, which is characterized by production of antibodies (Stabel, 2000). Because of this long incubation period (McKenna et al., 2006), dairy cows are most likely to become ELISA positive years after initial natural MAP exposure (Nielsen and Ersbøll, 2006). Although humoral immunity can be manifest as early as 10 to 17 mo after experimental infection, testing before $2 \mathrm{yr}$ of age is typically not recommended (Lepper et al., 1989). However, recent studies have contradicted these findings. For example, IgG against purified MAP antigen was reported to detect subclinically infected cattle (Koets et al., 2001). An ELISA using lipoarabinomannan as antigen detected antibodies starting at approximately 4 mo after challenge; furthermore, using immunoblot analysis, antibodies were detected as early as 2 wk after challenge (Waters et al., 2003). Additionally, using specific antigens, an antibody response was detected as early as $70 \mathrm{~d}$ after MAP exposure (Bannantine et al., 2008).

Among tests for screening dairy herds, ELISA is the most widely used. Specificity and sensitivity of serum ELISA ranged from 7 to $100 \%$ and 7 to $94 \%$, respectively (Nielsen and Toft, 2008). This wide range in reported test characteristics was attributed to using various ELISA with specific antigen preparations and gold standards, stage of disease, and age distribution of tested animals (Nielsen and Toft, 2008). Additionally, ELISA has a higher sensitivity in high shedders compared with low shedders (Whitlock et al., 2000). Therefore, an ELISA performs best in older animals in high-prevalence herds (Kalis et al., 2002). Calves shed MAP for a 6-mo interval right after infection (Mitchell et al., 2012) in which they could also test ELISA positive. However, no large-scale, longitudinal experimental infection experiment has apparently been done to determine the production of MAP-specific antibodies using a commercial ELISA under varying inoculation 
conditions. The objective of the current study was to use a commercially available ELISA to identify differences in humoral immune responses early after infection, in calves experimentally infected with 2 doses of MAP at 5 ages.

\section{MATERIALS AND METHODS}

\section{Herds and Calves}

The study design and sample collection have already been described (Mortier et al., 2013). Briefly, calves were collected from low-prevalence herds $(<5 \%$ seropositive) in Southern Alberta (Canada) and included in the study when born in the presence of a member of our research team. All dams were negative on a MAP ELISA (Idexx Paratuberculosis Ab Test; Idexx Laboratories Inc., Westbrook, ME) and fecal culture. Furthermore, all calves in this study tested negative for bovine viral diarrhea antigen (samples processed by Feedlot Health Management Services, Okotoks, AB, Canada).

Upon arrival at the research facility, calves were fed $6 \mathrm{~L}$ of $\gamma$-irradiated colostrum within $6 \mathrm{~h}$ after birth. The colostrum used in this study was collected from ELISA-negative herds to ensure the absence of MAPspecific antibodies. This colostrum was then treated with $\gamma$ irradiation, with a minimum dose of $10 \mathrm{kGy}$ per pail (containing $17 \mathrm{~L}$ of colostrum) using a Co60 source (McMaster Nuclear Reactor, Hamilton, ON, Canada; Garin-Bastuji et al., 1990) to ensure it did not contain any live MAP bacteria. The calves were then fed milk replacer (Grober Nutrition milk replacer; Grober Nutrition, Airdrie, AB, Canada), calf starter grain (without antimicrobial additives; Feed-Rite, Winnipeg, MB, Canada), and high-quality hay.

Calves were individually housed under stringent biosecurity conditions. The calves were monitored until 17 mo of age ( + or $-2 \mathrm{wk})$. Consequently, calves inoculated at 2 wk or at $3,6,9$, or 12 mo were followed for $17,14,11,8$, or 5 mo after inoculation, respectively. Health status was monitored on a daily basis by clinical inspection. At 17 mo of age, euthanasia and necropsies were performed, including assessment of gross and histological lesions as well as tissue culture (Mortier et al., 2013). Animal care protocols M09083 and M09050 were reviewed and approved by the Animal Care Committee of the University of Calgary (Calgary, AB, Canada).

\section{Study Design}

Upon arrival at the research facility, 50 calves were randomly allocated to 5 groups that would be orally challenged at various ages (2 wk or 3, 6, 9, and $12 \mathrm{mo}$ ). An additional 6 calves housed in the same conditions were not inoculated and served as negative controls. Within each of the 5 age groups containing 10 calves, 5 calves were inoculated orally with a high dose (HD) of MAP and 5 calves were inoculated with a low dose (LD) of MAP.

The maximum capacity of the research facility was 33 calves housed individually. Consequently, the first 33 calves equally representing all age and dose groups, as well as 3 controls were included. The experiment was then repeated with 23 calves, including 3 control calves, also equally representing all age and dose groups.

\section{Inoculum}

The inoculum preparation has been described by Mortier et al. (2013). Briefly, a virulent cattle type MAP strain isolated from a clinical Alberta JD case (cow 69) was used for inoculation. This isolate has an identical BamHI, PvuII, and PstI IS900 RFLP profile as the reference strain K10 (Hines et al., 2007). Calves were challenged orally on 2 consecutive days, with either $5 \times 10^{9} \mathrm{cfu}(\mathrm{HD})$ or $5 \times 10^{7} \mathrm{cfu}(\mathrm{LD})$. The inoculum was prepared in 1 batch and cultured in 7H9 broth. It was quantified using the pelleted wet-weight method as well as quantitative PCR, after which it was stored at -80 until used in the trial. Before each inoculation, 1 tube containing an identical aliquot of MAP cells was taken out of the $-80^{\circ} \mathrm{C}$ freezer and resuspended in $350 \mathrm{~mL}$ of $7 \mathrm{H} 9 \mathrm{broth}$. The culture was incubated for exactly $7 \mathrm{~d}$ at $37^{\circ} \mathrm{C}$ in a shaking incubator. In this period, the inoculum was tested for contamination. Immediately before inoculation, a 50-mL volume was prepared for the HD inoculation group and a 100-fold dilution was created for the LD inoculation group. The inoculum was placed in a syringe and expelled at the root of the tongue.

\section{Sampling and ELISA}

Serum samples were collected before inoculation. During the first month after infection, serum samples were collected weekly and from the second month to necropsy, serum samples were taken monthly.

Serum samples were analyzed for MAP-specific antibodies using the Idexx Paratuberculosis Ab Test (Idexx Laboratories Inc.) according to the manufacturer's instructions. However, 1 modification was made regarding interpretation of the test. Results of the test sample were expressed as a proportion of the positive control, corrected for the negative control [sample-to-positive $(\mathbf{S} / \mathbf{P})$ ratio], thereby eliminating interplate variation. An S/P ratio of 60 was considered ELISA positive. According to the manufacturer's description, an $\mathrm{S} / \mathrm{P}$ ratio of 60 to 70 should be considered as suspicious when testing an animal in a herd a single time. However, be- 
Table 1. Number and percentage (in parentheses) of calves in each group with at least 1 seropositive result ${ }^{1}$

\begin{tabular}{lllllll}
\hline Item & $2 \mathrm{wk}$ & $3 \mathrm{mo}$ & $6 \mathrm{mo}$ & 9 mo & 12 mo & Total \\
\hline High dose & $4(80)$ & $3(60)$ & $2(40)$ & $2(40)$ & $1(20)$ & $13(48)$ \\
Low dose & $2(40)$ & $2(40)$ & $0(0)$ & $3(60)$ & $2(40)$ & $9(36)$ \\
Total & $6(60)$ & $5(50)$ & $2(20)$ & $5(50)$ & $3(30)$ & \\
\hline
\end{tabular}

${ }^{1}$ Calves were assigned to 10 groups and inoculated with a high or low dose of Mycobacterium avium ssp. paratuberculosis (MAP) at 2 wk or $3,6,9$, or 12 mo of age.

cause calves in this study were experimentally infected, the authors considered suspicious calves as positive. Observed antibody profile types in this study were described as "persistent response," "peak response," and "bimodal peak response" (Kawaji et al., 2012).

\section{Statistical Analyses}

All statistical analyses were performed using STATA 11 software (StataCorp LP, College Station, TX). A Pvalue $<0.05$ was considered significant. Tissue culture results and macroscopic and microscopic lesions were assigned to categories (Mortier et al., 2013). The ELISA results were categorized as follows: $0=$ no ELISApositive samples and $1=$ ELISA positive at least once after inoculation and before necropsy. Differences in distributions of tissue culture results and macroscopic and microscopic lesions between ELISA were evaluated using chi-squared and Fisher exact tests.

The magnitude of the serological response was analyzed using the area under the curve (AUC) of the S/P ratio for the first $4.5 \mathrm{mo}$ after inoculation; this was done to ensure the same number of observations for each group (the last group was inoculated at 12 mo of age and all calves were euthanized as 17 mo of age). The AUC was compared across age and dose groups using the Kruskal-Wallis test (Dupont, 2009) to identify differences in antibody responses between age and dose groups. To correct for multiple comparisons, a $P$-value of 0.003 was implemented using the following formula: $\alpha=\alpha /[2 \times(\kappa-1)]$, where $\alpha$ is the significance and $\kappa$ is the number of observations (Dupont, 2009). To evaluate development of MAP antibodies over a longer interval (9 mo), a separate analysis was conducted excluding calves inoculated at 9 and 12 mo of age. The adjusted $P$-value for this analysis was 0.025 .

\section{RESULTS}

A humoral immune response was detected at least once during the entire follow-up period in $21(42 \%)$ of the 50 MAP-inoculated calves [Table 1; Figure 1; Supplementary Tables S1-S6 (http://dx.doi.org/10.3168/ jds.2014-8139)]. All control calves remained ELISA negative during the course of the trial (Supplementary
Table S6). Calves inoculated at 2 wk or at 3, 6, 9, or 12 mo were followed for $17,14,11,8$, and 5 mo after inoculation, respectively, except for 2 calves from the 2-wk-HD group that developed clinical symptoms indicative of JD and were euthanized at 16 mo of age.

Enzyme-linked immunosorbent assay-positive calves existed in all age and dose groups except the 6-mo-LD group [Figure 1; Supplementary Tables S1-S6 (http:// dx.doi.org/10.3168/jds.2014-8139)]. The greatest number of calves with at least 1 positive sample was in the 2 -wk inoculation group (6 of 10 calves positive), with 4 of $5(80 \%)$ calves of the HD group ELISA positive compared with 2 of $5(40 \%)$ from the LD group (Table $1)$. Twelve of $25(48 \%)$ calves inoculated with an HD of MAP were ELISA positive at least once, compared with 9 of $25(36 \%)$ inoculated with a LD of MAP (positive at least once; Table $1 ; P=0.39$ ).

Starting at 4 mo after inoculation, at any sampling, HD calves were more frequent seropositive compared with LD calves (apparent from Figures 1 and 2). This was predominantly caused by the calves inoculated at 2 wk and 3 mo of age (Figure 1).

The "persistent response" was observed 5 times (for example, 3 times in the 2 -wk-HD group; Figure 1). The "peak response" was observed 7 times (for example, in the 6-mo-HD group; Figure 1), whereas a "bimodal peak response" was observed once in the 3-mo-HD group (Figure 1). In 7 cases, it was unclear which response profile the calf would develop, due to completion of the trial (for example, in the 2-wk-LD group; Figure 1).

Calves with at least 1 positive ELISA sample had no higher or lower chance than ELISA-negative calves of being positive grossly, histologically, or on tissue culture $(P=0.20,0.29$, and 0.35 , respectively). The 2 calves with clinical symptoms of JD both became ELISA positive at 4 and 5 mo after inoculation. These 2 calves also had several positive tissue locations and high histology and macroscopy scores $(P=0.003,0.001$, and 0.002 , respectively; Table 2 ).

When comparing the groups for the first 4.5 mo after inoculation, calves inoculated at 12 mo with an $\mathrm{HD}$ or an LD had a larger AUC of the ELISA S/P ratio, indicating a stronger initial serological response, than the 2 -wk-LD group ( $P=0.001$ and 0.0004 , respectively). The other groups were not different compared with the 


\section{Low dose}

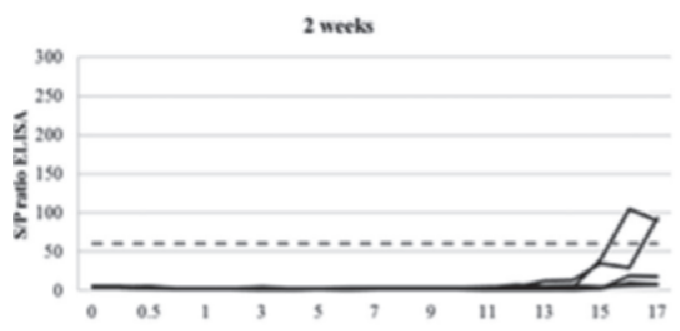

3 months

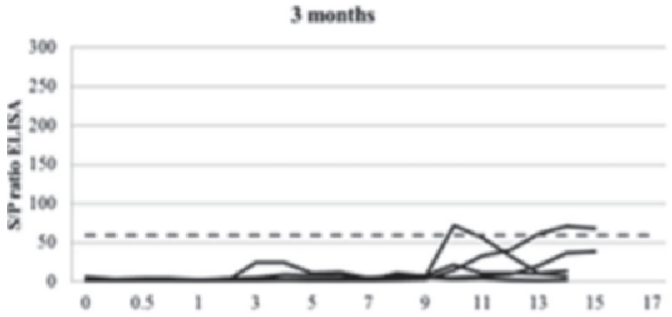

6 months

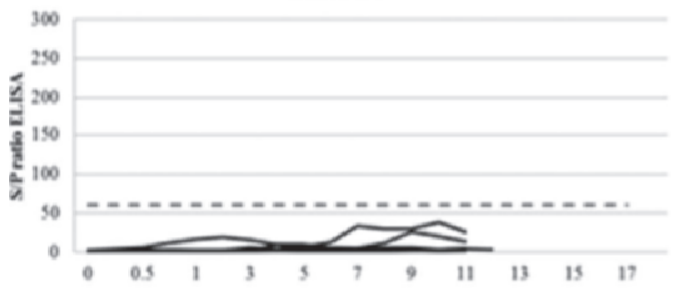

9 months

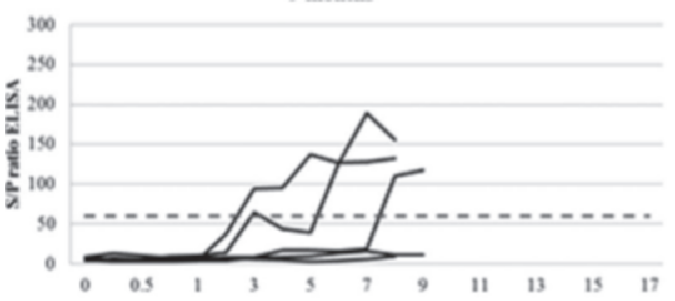

12 months

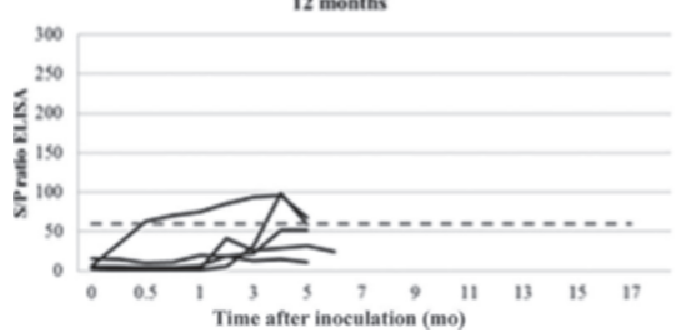

High dose

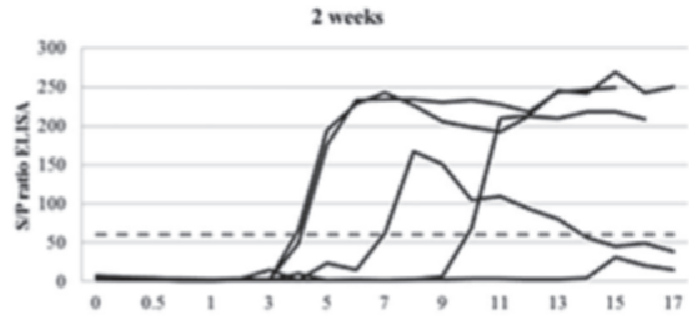

3 months

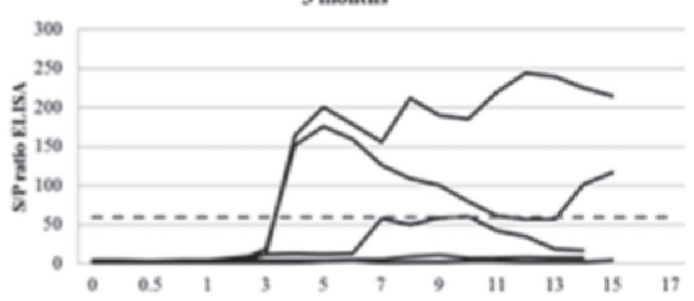

6 months

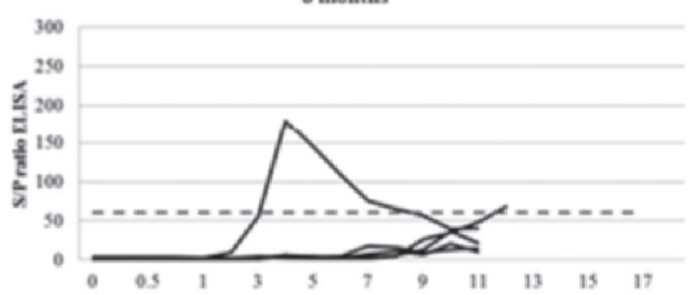

9 months

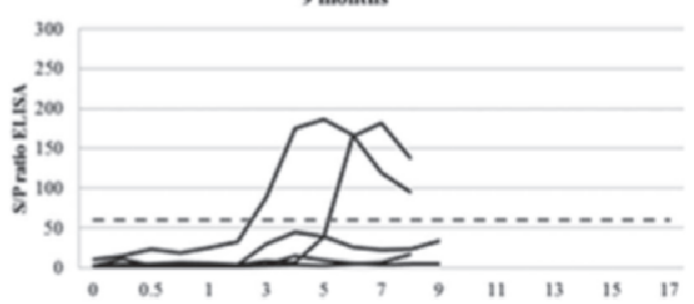

12 months

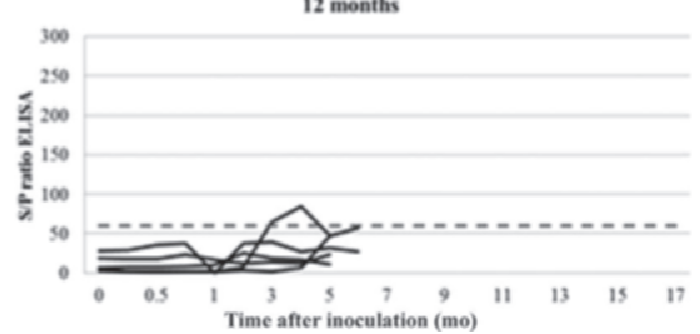

Figure 1. The ELISA sample-to-positive (S/P) ratio in 10 inoculation groups for each individual calf. Calves were inoculated with a high or low dose of Mycobacterium avium ssp. paratuberculosis (MAP) at 2 wk or $3,6,9$, or 12 mo of age. Samples with a cutoff $>60$ (dashed line) are considered positive.

2-wk-LD group (lowest $P$-value $=0.004$; the adjusted $P$-value for significance for these multiple comparisons was 0.003). After exclusion of the 9- and 12-moinoculation groups and analyzing the AUC for a 9-mo interval, the 2-wk-HD, 3-mo-HD, and 6-mo-HD calves had a larger AUC than the 2 -wk-LD group $(P=0.001$, 0.004 , and 0.006 , respectively; the adjusted $P$-value for significance for these multiple comparisons was 0.025 ). 
Table 2. Comparison of ELISA and necropsy findings in 50 calves experimentally infected at 5 ages using 2 doses of Mycobacterium avium ssp. paratuberculosis (MAP)

\begin{tabular}{|c|c|c|c|c|c|c|c|c|c|c|c|c|c|}
\hline \multirow[b]{2}{*}{ ELISA $^{1}$} & \multicolumn{4}{|c|}{ Tissue culture $^{2}$} & \multicolumn{4}{|c|}{ Histology ${ }^{3}$} & \multicolumn{5}{|c|}{ Macroscopy $^{4}$} \\
\hline & 0 & 1 & 2 & 3 & 0 & 1 & 2 & 3 & 0 & 1 & 2 & 3 & 4 \\
\hline 0 & 13 & 14 & 2 & 0 & 5 & 22 & 2 & 0 & 12 & 2 & 2 & 13 & 0 \\
\hline 1 & 9 & 9 & 1 & $2^{5}$ & 3 & 14 & 2 & $2^{5}$ & 7 & 2 & 0 & 10 & $2^{5}$ \\
\hline \multicolumn{14}{|c|}{$\begin{array}{l}{ }^{1} 0=\text { no ELISA-positive samples; } 1=\text { ELISA-positive at least once during the period after inoculation and } \\
\text { before necropsy. }\end{array}$} \\
\hline \multirow{2}{*}{\multicolumn{14}{|c|}{$\begin{array}{l}{ }^{2} 0=\text { no tissue locations culture positive; } 1=1 \text { to } 3 \text { tissue locations culture positive; } 2=4 \text { to } 6 \text { tissue locations } \\
\text { culture positive; } 3=>6 \text { tissue locations culture positive. } \\
{ }^{3} 0=\text { no lesions; } 1=\text { focal lesions; } 2=\text { multifocal lesions; } 3=\text { diffuse lymphocytic, multibacillary, or intermedi- } \\
\text { ate lesions. }\end{array}$}} \\
\hline & & & & & & & & & & & & & \\
\hline \multicolumn{14}{|c|}{$\begin{array}{l}{ }^{4} 0=\text { no macroscopic changes; } 1=1 \text { enlarged or edematous lymph node of the small intestine or liver; } 2= \\
\text { multiple enlarged and edematous mesenteric lymph nodes or hyperemia of the ileocecal valve, or both; } 3= \\
\text { enlarged mesenteric lymph node(s) and (or) mild to moderate thickening of ileal or jejunal mucosa; } 4=\text { en- } \\
\text { larged mesenteric lymph node(s) and severe thickening and corrugation of the ileal, jejunal, and colon mucosa. }\end{array}$} \\
\hline
\end{tabular}

\section{DISCUSSION}

A serological response against MAP was detected in all age and dose groups, except for calves infected at $6 \mathrm{mo}$ of age with an LD. Calves inoculated with an HD had a larger AUC and, thus, a stronger antibody response, particularly when inoculated at a younger age ( 2 wk or 3 or 6 mo). Moreover, the humoral immune response started before 4.5 mo after inoculation in calves inoculated at an older age (12 mo).

An antibody response was detected in calves early after infection (Figure 1), consistent with other studies
(Koets et al., 2001; Bannantine et al., 2008); however, it was noteworthy that the previous studies did not use a commercially available ELISA. To increase consistency and applicability, it is desirable to use an ELISA that is readily available and validated, and used in commercial labs that are available to practitioners and producers. Although a serological response was detected in only $42 \%$ of the orally inoculated calves in the current study, it is noteworthy that more calves tested positive than expected. This can complement knowledge suggesting that ELISA is most useful in naturally exposed adults (Lepper et al., 1989; Kalis et al., 2002; Nielsen and

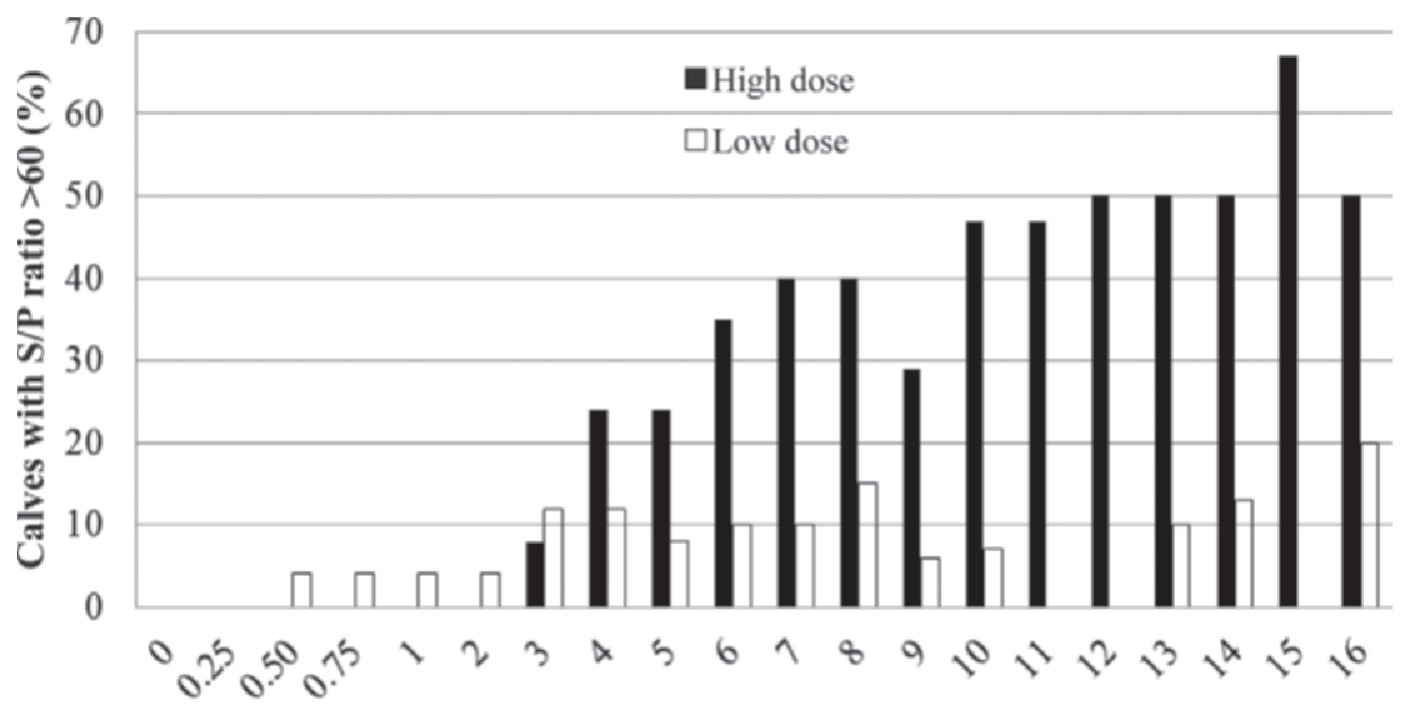

Time after inoculation (mo)

Figure 2. Percentage of calves with a sample-to-positive $(\mathrm{S} / \mathrm{P})$ ratio $>60$ in the high- and low-dose groups for every month after inoculation, including all 5 inoculation ages. The proportion of positive calves of a total number of calves at each time point (x-axis) is plotted for the high-dose (HD) as well and the low-dose (LD) group. Note: the number of calves used to calculate this proportion decreased as the time after inoculation increased (as calves inoculated at $2 \mathrm{wk}$ or at 3,6, 9, or 12 mo were followed for 17, 14, 11, 8, or 5 mo after inoculation, respectively). 
Ersbøll, 2006; Nielsen and Toft, 2008); using ELISA might also be valuable in cattle younger than 2 yr.

The humoral immune response was clearly dose dependent; in calves inoculated at 2 wk or 3 or 6 mo, fewer LD than HD calves became ELISA positive. Consequently, calves exposed to an LD of MAP on farm might not mount a detectable antibody response. These findings were consistent with previous reports that earlier antibody production is consistent with higher initial doses of MAP (Waters et al., 2003; Coussens, 2004). An inoculation dose was selected for the current study that would mimic ingestion of MAP occurring naturally on a farm. Infection occurred in young calves with a dose contained in $2 \mathrm{~g}$ of infected feces (McKenna et al., 2006). However, the number of colony-forming units present in these $2 \mathrm{~g}$ remains to be determined. A dose of $1.5 \times 10^{6} \mathrm{cfu}$ reliably produced infection in young calves (Sweeney et al., 2006), based on which an LD of $5 \times 10^{7}$ cfu was chosen. An HD that was a 100 -fold higher $\left(5 \times 10^{9} \mathrm{cfu}\right)$ was then selected based on the assumption that a larger dose would be needed to infect older animals; furthermore, this dose was previously recommended for experimental infection studies (Hines et al., 2007). In the current study, 2 calves inoculated with an HD of MAP at 2 wk of age had clinical symptoms of JD. The question remains whether these calves were inherently more susceptible to infection than the other 3 calves in the 2 -wk-HD group. This early manifestation of clinical symptoms is atypical of JD (Hines et al., 2007) and could indicate that the challenge dose was too high. On the other hand, $20 \%$ of heifers in high-prevalence herds were shedding before 2 yr of age (Weber et al., 2010), indicating that even young animals are shedding under high infection pressure and clinical disease in young animals might be underreported because parasitic infections and other causes of diarrhea are thought of before JD.

In mouse models, it was observed that the antigen dose can direct the immune response towards a Th1 or Th2 response. In a review on this topic, it was summarized that very low and high antigen doses favored Th1 responses, whereas intermediate-high doses favored Th2 (Golding and Scott, 1995). However, conflicting reports were presented in this review in relation to whether Th1 or Th2 responses are caused by high or low doses of antigen (Constant and Bottomly, 1997). The antigen (bacteria vs. parasites) itself can affect the generated immune response as well as the available number of antigenic epitopes during T-cell priming (Constant and Bottomly, 1997). It is noteworthy that in vitro experiments and murine systems using various pathogens do not compare well with MAP infection trials in cattle; therefore, additional research is needed. Because MAP suppresses antigen presentation (Weiss et al., 2001;
Purdie et al., 2012), we inferred that a higher dose of MAP would cause more suppression. In contrast, in the present study, a greater antibody response occurred in calves inoculated with an HD of MAP. Presumably, suppression was absent or ineffective, or perhaps overruled by other factors. Additionally, for unknown reasons, this difference in response between an HD and an LD of MAP disappeared when inoculated at 9 or 12 mo of age. Also, in studies focused on gene expression profiling, differences existed between infected and noninfected animals in major histocompatibility complex gene modulation, playing a crucial role in antigen presentation to T cells (Purdie et al., 2012), which might provide insights into the dose-dependent humoral immune responses.

Calves inoculated at $2 \mathrm{wk}$ or 3 mo of age had a lower AUC in the first 4.5 mo after inoculation compared with the 12-mo group. Therefore, calves inoculated at 12 mo of age started producing antibodies sooner after inoculation compared with calves in the 2 -wk or 3 -mo groups. In the 12-mo inoculation group, 1 calf even became seropositive at $2 \mathrm{wk}$ after inoculation. This was in agreement with evidence that humoral immunity occurred early after infection and can be detected using specific antigens (Kawaji et al., 2012). A possible explanation for this later onset of antibody production is an inherent inability to mount an active immune response in young calves (Chase et al., 2008). Although all essential immune components identified in adults are present in the neonate at birth, they are incapable of full function for the first month after birth (Reber et al., 2006; Chase et al., 2008). The proportion of B-lymphocytes is low at birth and takes some time to increase to adult levels (Chase et al., 2008). It is, therefore, likely that neonatal calves have low antibody-producing capacity by lymphocytes until 1 mo after birth (Nagahata et al., 1991). The highest suppressor activity by $\gamma \delta \mathrm{T}$ cells is registered at birth and decreases to adult levels later on (Wilson et al., 1996), again delaying a humoral immune response. Additionally, presence of leukocytes in colostrum ensures faster development of a cellular immune response (Reber et al., 2005). In the current study, however, colostrum was $\gamma$ irradiated and these maternal leukocytes were destroyed; therefore, the active humoral response could have been delayed. It is noteworthy that none of the calves in this study had failure of transfer of maternal immunity.

Calves with at least 1 positive ELISA sample did not have a higher or lower chance of being positive for gross lesions, histologically or on tissue culture. However, the 2 calves with clinical symptoms of JD that had severe necropsy lesions and positive tissue culture became ELISA positive 4 and 5 mo after inoculation and remained positive until necropsy. In previous re- 
ports, a low sensitivity of ELISA existed when using tissue culture as a gold standard (9-17\%; McKenna et al., 2005). Additionally, it was reported that ELISA performance varied with the various stages of JD. In clinically affected cattle, ELISA had a higher sensitivity (Nielsen and Toft, 2008) and, thus, a higher association can be expected between ELISA and necropsy lesions and tissue culture in cattle with MAP infection that has progressed to the clinical stage.

Antibody response profiles were identified to characterize antibody responses in experimentally infected calves (Kawaji et al., 2012). The same 3 distinct types of antibody response profiles were observed in the current study: transient "peak responses," "bimodal peak response," and "persistent responses" throughout the different age and dose groups (Figure 1). Remarkably, great individual variability existed within groups in responses observed in the current study, consistent with previous reports (Muskens et al., 2002; Mortensen et al., 2004; Kawaji et al., 2012) as well as the transience in some of these responses. Not only the performance of the ELISA needs to be taken into account when screening a herd for MAP infection, but also differences in antibody response profile between individual animals.

\section{CONCLUSIONS}

Mycobacterium avium ssp. paratuberculosis infection can be detected earlier than originally expected using a commercially available ELISA; therefore, screening young stock in combination with adult cattle might be useful in the context of JD control programs. In contrast, calves exposed to an LD of MAP, which could occur in low-prevalence herds, might be missed using a herd-screening ELISA. Additionally, transient responses and individual variations in antibody responses should be taken into account, because these might also lead to incorrect interpretation of an ELISA result.

\section{ACKNOWLEDGMENTS}

This work was supported by Alberta Innovates Bio Solutions (Edmonton, AB, Canada), the Alberta Livestock and Meat Agency (Edmonton, AB, Canada), Alberta Milk (Edmonton, AB, Canada), Dairy Farmers of Canada (Ottawa, ON, Canada), the Natural Sciences and Engineering Research Council of Canada (Ottawa, ON, Canada), and University of Calgary, Faculty of Veterinary Medicine (Calgary, AB, Canada). The authors thank Amanda Nicol, Sandeep Atwal, Uliana Kanevets, Alicia Parfett, Erin Vernooy, Michelle Drissler, Lee Head, and Gwendolyn Roy (all from Department of Production Animal Health, University of Calgary, Calgary, AB, Canada) for technical assistance.
The authors also acknowledge numerous undergraduate students that have collaborated in this trial and John Kastelic (Department of Production Animal Health, University of Calgary) for editing the manuscript.

\section{REFERENCES}

Bannantine, J. P., D. O. Bayles, W. R. Waters, M. V. Palmer, J. R. Stabel, and M. L. Paustian. 2008. Early antibody response against Mycobacterium avium subspecies paratuberculosis antigens in subclinical cattle. Proteome Sci. 6:5.

Benedictus, G., A. A. Dijkhuizen, and J. Stelwagen. 1987. Economic losses due to paratuberculosis in dairy cattle. Vet. Rec. 121:142146.

Chase, C. C., D. J. Hurley, and A. J. Reber. 2008. Neonatal immune development in the calf and its impact on vaccine response. Vet. Clin. North Am. Food Anim. Pract. 24:87-104.

Chi, J., J. A. VanLeeuwen, A. Weersink, and G. P. Keefe. 2002. Direct production losses and treatment costs from bovine viral diarrhoea virus, bovine leukosis virus, Mycobacterium avium subspecies paratuberculosis, and Neospora caninum. Prev. Vet. Med. 55:137-153.

Constant, S. L., and K. Bottomly. 1997. Induction of Th1 and Th2 $\mathrm{CD}^{+} \mathrm{T}$ cell responses: The alternative approaches. Annu. Rev. Immunol. 15:297-322.

Coussens, P. M. 2004. Model for immune responses to Mycobacterium avium subspecies paratuberculosis in cattle. Infect. Immun. 72:3089-3096.

Dupont, W. D. 2009. Repeated-measures analysis of variance. Pages 451-483 in Statistical Modeling for Biomedical Researchers: A Simple Introduction to the Analysis of Complex Data. Cambridge University Press, Cambridge, UK.

Garin-Bastuji, B., B. Perrin, M.-F. Thorel, and J. L. Martel. 1990. Evaluation of $\gamma$-rays irradiation of cows' colostrum for Brucella abortus, Escherichia coli K99, Salmonella dublin and Mycobacterium paratuberculosis decontamination. Lett. Appl. Microbiol. 11:163-166.

Golding, B., and D. E. Scott. 1995. Vaccine strategies: Targeting helper T cell responses. Ann. N. Y. Acad. Sci. 754:126-137.

Hines, M. E., II, J. R. Stabel, R. W. Sweeney, F. Griffin, A. M. Talaat, D. Bakker, G. Benedictus, W. C. Davis, G. W. de Lisle, I. A. Gardner, R. A. Juste, V. Kapur, A. Koets, J. McNair, G. Pruitt, and R. H. Whitlock. 2007. Experimental challenge models for Johne's disease: A review and proposed international guidelines. Vet. Microbiol. 122:197-222.

Kalis, C. H. J., H. W. Barkema, J. W. Hesselink, C. van Maanen, and M. T. Collins. 2002. Evaluation of two absorbed enzyme-linked immunosorbent assays and a complement fixation test as replacements for fecal culture in the detection of cows shedding Mycobacterium avium subspecies paratuberculosis. J. Vet. Diagn. Invest. 14:219-224.

Kawaji, S., R. Nagata, R. J. Whittington, and Y. Mori. 2012. Detection of antibody responses against Mycobacterium avium subsp. paratuberculosis stress-associated proteins within 30 weeks after infection in cattle. Vet. Immunol. Immunopathol. 150:101-111.

Koets, A. P., V. P. Rutten, M. de Boer, D. Bakker, P. ValentinWeigand, and W. van Eden. 2001. Differential changes in heat shock protein-, lipoarabinomannan-, and purified protein derivative-specific immunoglobulin G1 and G2 isotype responses during bovine Mycobacterium avium subsp. paratuberculosis infection. Infect. Immun. 69:1492-1498.

Kudahl, A., S. S. Nielsen, and J. T. Sørensen. 2004. Relationship between antibodies against Mycobacterium avium subsp. paratuberculosis in milk and shape of lactation curves. Prev. Vet. Med. 62:119-134.

Lepper, A. W. D., C. R. Wilks, M. Kotiw, J. T. Whitehead, and K. S. Swart. 1989. Sequential bacteriological observations in relation to cell-mediated and humoral antibody responses of cattle infected with Mycobacterium paratuberculosis and maintained on normal or high iron intake. Aust. Vet. J. 66:50-55. 
McKenna, S. L. B., G. P. Keefe, D. C. Sockett, and H. W. Barkema. 2005. Evaluation of three ELISAs for Mycobacterium avium subsp. paratuberculosis using tissue and fecal culture as comparison standards. Vet. Microbiol. 110:105-111.

McKenna, S. L. B., G. P. Keefe, A. Tiwari, J. VanLeeuwen, and H. W. Barkema. 2006. Johne's disease in Canada part II: Disease impacts, risk factors, and control programs for dairy producers. Can. Vet. J. 47:1089-1099.

Mitchell, R. M., G. F. Medley, M. T. Collins, and Y. H. Schukken. 2012. A meta-analysis of the effect of dose and age at exposure on shedding of Mycobacterium avium subspecies paratuberculosis (MAP) in experimentally infected calves and cows. Epidemiol. Infect. 140:231-246.

Mortensen, H., S. S. Nielsen, and P. Berg. 2004. Genetic variation and heritability of the antibody response to Mycobacterium avium subspecies paratuberculosis in Danish Holstein cows. J. Dairy Sci. $87: 2108-2113$.

Mortier, R. A. R., H. W. Barkema, J. M. Bystrom, O. Illanes, K. Orsel, R. Wolf, G. Atkins, and J. De Buck. 2013. Evaluation of age-dependent susceptibility in calves infected with two doses of Mycobacterium avium subspecies paratuberculosis using pathology and tissue culture. Vet. Res. 44:94.

Muskens, J., F. van Zijderveld, A. Eger, and D. Bakker. 2002. Evaluation of the long-term immune response in cattle after vaccination against paratuberculosis in two Dutch dairy herds. Vet. Microbiol. 86:269-278.

Nagahata, H., N. Kojima, I. Higashitani, H. Ogawa, and H. Noda. 1991. Postnatal changes in lymphocyte function of dairy calves. Zentralbl. Veterinärmed. B 38:49-54.

Nielsen, S. S., and A. K. Ersbøll. 2006. Age at occurrence of Mycobacterium avium subspecies paratuberculosis in naturally infected dairy cows. J. Dairy Sci. 89:4557-4566.

Nielsen, S. S., and N. Toft. 2008. Ante mortem diagnosis of paratuberculosis: A review of accuracies of ELISA, interferon-gamma assay and faecal culture techniques. Vet. Microbiol. 129:217-235.

Purdie, A. C., K. M. Plain, D. J. Begg, K. de Silva, and R. J. Whittington. 2012. Expression of genes associated with the antigen presentation and processing pathway are consistently regulated in early Mycobacterium avium subsp. paratuberculosis infection. Comp. Immunol. Microbiol. Infect. Dis. 35:151-162.

Reber, A. J., A. R. Hippen, and D. J. Hurley. 2005. Effects of the ingestion of whole colostrum or cell-free colostrum on the capacity of leukocytes in newborn calves to stimulate or respond in one-way mixed leukocyte cultures. Am. J. Vet. Res. 66:1854-1860.
Reber, A. J., A. Lockwood, A. R. Hippen, and D. J. Hurley. 2006. Colostrum induced phenotypic and trafficking changes in maternal mononuclear cells in a peripheral blood leukocyte model for study of leukocyte transfer to the neonatal calf. Vet. Immunol. Immunopathol. 109:139-150.

Stabel, J. R. 2000. Transitions in immune responses to Mycobacterium paratuberculosis. Vet. Microbiol. 77:465-473.

Sweeney, R. W., J. Uzonna, R. H. Whitlock, P. L. Habecker, P. Chilton, and P. Scott. 2006. Tissue predilection sites and effect of dose on Mycobacterium avium subs. paratuberculosis organism recovery in a short-term bovine experimental oral infection model. Res. Vet. Sci. 80:253-259.

Tiwari, A., J. A. VanLeeuwen, S. L. B. McKenna, G. P. Keefe, and H. W. Barkema. 2006. Johne's disease in Canada: Part I: Clinical symptoms, pathophysiology, diagnosis, and prevalence in dairy herds. Can. Vet. J. 47:874-882.

Waters, W. R., J. M. Miller, M. V. Palmer, J. R. Stabel, D. E. Jones, K. A. Koistinen, E. M. Steadham, M. J. Hamilton, W. C. Davis, and J. P. Bannantine. 2003. Early induction of humoral and cellular immune responses during experimental Mycobacterium avium subsp. paratuberculosis infection of calves. Infect. Immun. 71:5130-5138

Weber, M. F., J. Kogut, J. de Bree, G. van Schaik, and M. Nielen. 2010. Age at which dairy cattle become Mycobacterium avium ssp. paratuberculosis faecal culture positive. Prev. Vet. Med. 97:29-36.

Weiss, D. J., O. A. Evanson, D. J. McClenahan, M. S. Abrahamsen, and B. K. Walcheck. 2001. Regulation of expression of major histocompatibility antigens by bovine macrophages infected with $\mathrm{Myco-}$ bacterium avium subsp. paratuberculosis or Mycobacterium avium subsp. avium. Infect. Immun. 69:1002-1008.

Whitlock, R. H., and C. Buergelt. 1996. Preclinical and clinical manifestations of paratuberculosis (including pathology). Vet. Clin. North Am. Food Anim. Pract. 12:345-356.

Whitlock, R. H., S. J. Wells, R. W. Sweeney, and J. Van Tiem. 2000. ELISA and fecal culture for paratuberculosis (Johne's disease): Sensitivity and specificity of each method. Vet. Microbiol. $77: 387-398$.

Wilson, R. A., A. Zolnai, P. Rudas, and L. V. Frenyo. 1996. T-cell subsets in blood and lymphoid tissues obtained from fetal calves, maturing calves and adult bovine. Vet. Immunol. Immunopathol. 53:49-60. 